\title{
Treatment responses in multidrug-resistant tuberculosis in Germany
}

4
${ }^{1}$ Division of Clinical Infectious Diseases, Research Center Borstel, Borstel, Germany ${ }^{2}$ German Center for Infection Research (DZIF), Germany ${ }^{3}$ International Health/Infectious Diseases, University of Lübeck, Lübeck, Germany ${ }^{4}$ Department of Global Health, Academic Medical Center, University of Amsterdam, Amsterdam Institute for Global Health and Development, Amsterdam

${ }^{5}$ Asklepios Fachkliniken München-Gauting, Munich, Germany

${ }^{6}$ Karl-Hansen-Klinik, Bad Lippspringe, Germany

${ }^{7}$ Department of Medicine, University of Namibia School of Medicine, Windhoek, Namibia ${ }^{8}$ Sankt Katharinen-Krankenhaus, Frankfurt, Germany

${ }^{9}$ Sections of Infectious Diseases and Tropical Medicine, I. Department of Internal Medicine, University Medical Centre Hamburg-Eppendorf, Hamburg, Germany

${ }^{10} \mathrm{Clinical}$ Research Unit, Bernhard-Nocht-Institute for Tropical Medicine, Hamburg-Germany

${ }^{11}$ Thoraxklinik-Heidelberg, Heidelberg, Germany

${ }^{12}$ Department of Medicine, Karolinska Institute, Stockholm, Sweden

*Corresponding author: Dr. Jan Heyckendorf, Clinical Infectious Diseases, Medical Clinic, Research Center Borstel, Parkallee 35, 23845 Borstel, Germany; T+49 4537188 2901; Email: jheyckendorf@fz-borstel.de

Running head: Treatment Outcomes in M/XDR-TB

Keywords: MDR-TB, smear conversion, culture conversion, XDR-TB, tuberculosis Word count: 2223 words / Display items: 3 figures, 4 tables, 1 supplement table References: 38 
34 Recently, excellent treatment outcomes have been reported for patients with 35 multidrug/extensively drug-resistant tuberculosis (M/XDR-TB) in settings where optimal 36 resources for individualized therapy are available. We ascertained whether differences in 37 treatment responses still exist in patients with M/XDR-TB compared to patients with non$38 \mathrm{M} / \mathrm{XDR}-\mathrm{TB}$.

Patients with tuberculosis were prospectively enrolled between March 2013 and March 2016 at five hospitals in Germany. Treatment was conducted following current guidelines

41 and individualized on the basis of comprehensive drug-resistance testing. Two-months and 6-months sputum-smear and sputum-culture conversion rates were assessed. A clinical and a radiological score were used to assess the response to anti-tuberculosis therapy. Non-M/XDR-TB $(n=29)$ and M/XDR-TB $(n=46)$ patients showed similar rates of microbiological conversion (2-months smear-conversion-rate $90 \%$ vs. $78 \%$ and cultureconversion-rate $67 \%$ versus $61 \%$, respectively; time-to-smear/culture-conversion 19 (IQR1032) vs. 31 (IQR14-56) ( $p=0.066)$, and 39 (IQR17-67) vs. 39 (IQR6-85) days ( $p=0.191)$, respectively). Both clinical and radiological scores declined after the introduction of anti49 tuberculosis therapy. There were no significant differences of scores between the groups until 6 months of therapy.

51 Under optimal clinical conditions with availability of novel diagnostics and a wide range of 52 therapeutic options for individualized therapy, patients with M/XDR-TB achieve 6 month 53 culture conversion rates that are compatible to patients with non-M/XDR-TB. 


\section{Introduction}

55 Tuberculosis (TB) is the leading cause of mortality attributed to a single microbial pathogen worldwide $(1,2)$. The World Health Organization (WHO) estimates that more than 10 million people developed active TB in 2016, the highest ever-estimated number of affected patients in history. The emergence of multidrug-resistant (MDR; defined by bacillary resistance against rifampicin and isoniazid) and extensively drug-resistant (XDR; MDR plus resistance against at least one fluoroquinolone and one second-line injectable drug) TB is especially worrisome. M/XDR-TB has been related to high treatment costs, increased frequency of adverse drug-events, and poor therapy outcomes (1, 3-7). At the recent meeting of the G20 leaders in Hamburg, Germany, combatting antimicrobial resistance, including drug-resistant TB, has been identified as a global priority (8).

Currently, the World Health Organization (WHO) recommends a therapy duration for M/XDR-TB patients of at least 20 months unless specific criteria allow for a standardized short course MDR-TB regimen over 9-12 months (3, 9-13). Only approximately $50 \%$ of M/XDR-TB patients in Europe attain favorable outcomes. In contrast, in settings where individualized treatment can be provided, successful treatment outcomes are usually observed $(3,4,14-16)$. The concept of individualized therapy targets the special demands of every host and pathogen leading to tailored treatment in every patient (17). Currently, new diagnostic methods and novel drugs have been introduced that may improve treatment outcomes (17-20). 
Based on these observations, we aimed to compare 6-months culture conversion, as early indicators of treatment outcomes, in M/XDR-TB and non-M/XDR-TB patients from settings where optimal resources are available. Additionally, we evaluated a clinical and a radiological scoring system (21).

\section{Study population and methods}

Between March 2013 and March 2016 patients with TB identified by sputum GeneXpert MTB/RIF test (Cepheid, Sunnyvale, USA) were prospectively enrolled the Medical Clinic, Research Center Borstel; Karl-Hansen-Klinik, Bad Lippspringe; Sankt KatharinenKrankenhaus, Frankfurt; Thoraxklinik-Heidelberg, Heidelberg; Asklepios Fachkliniken München-Gauting, Munich in Germany. Patients with M/XDR-TB were enrolled consecutively at all centers after satisfying in- and exclusion criteria, and providing written informed consent. Patients with non-MDR-TB were also recruited if inclusion criteria were met or exclusion criteria were not met and they agreed with participation. Athough patients with non-MDR-TB were not strictly recruited consecutively at all centers, selection was not based on patients characteristics but depended on staff availability. Individuals were excluded if they were less than 18 years of age, not legally able to provide consent or if they were infected with HIV. All patients gave written informed consent. Following approval at the Ethics Committee of the University of Lübeck (AZ 12-233), Germany, the study protocol was approved at the local Ethic Committees of all participating centers.

Following rapid molecular identification of rifampicin resistance by GeneXpert, sputum samples underwent second-line molecular drug resistance testing of $M$. tuberculosis by lineprobe-assays (GenoType MTBDRplus and MTBDRs/ Hain Lifesciences, Nehren, Germany). 
100 Sputum samples also underwent phenotypic drug susceptibility testing according to WHO

101 recommendations at a certified and quality controlled microbiology laboratory (WHO

102 Supranational Reference Laboratory Network). During the in-patient period, sputum samples

103 were collected for smear microscopy and culture on a weekly basis. After discharge, sputum

104 was collected as part of routine follow-up visits. Demographic information was collected on 105 study enrolment.

107 A novel clinical score consisting of self-reported and objectively observed items (maximum

108 score of 30 points), which is based on a published scoring system (22), was recorded by a

109 physician during the clinical visits. Self-reported items were cough, hemoptysis, dyspnea,

110 thoracic pain, night sweats, loss of weight, and inability to walk (each one point). The

111 examined score items (if not indicated differently, one point each) consisted of axillary body

112 temperature $\left(>37^{\circ}=\right.$ one point, $>38^{\circ}=$ two points $)$, impaired consciousness, focal neurologic

113 deficits, body mass index $\left(\mathrm{kg} / \mathrm{m}^{2} ;<20=\right.$ one point, $<18=$ two points, $<16=$ three points),

114 middle upper arm circumference ( $\mathrm{mm} ;<220=$ one point, $<200=$ two points), capillary filling

115 time $>2$ sec., cyanosis, tachycardia (beats per minute; $>100=$ one point, $>120=$ two points),

116 blood pressure $<90 \mathrm{mmHg}$ systolic or $<60 \mathrm{mmHg}$ diastolic, lung crackles, tachypnea (per

117 minute; $>20=$ one point, $>25=$ two points, $>30=$ three points), oxygen saturation $(\%$; $<90=$

118 one point, $<87=2$ points), and age above 65 (one point).

120 Chest X-rays were performed at clinically relevant time-points during the course of

121 treatment. The extent of pulmonary TB was assessed by a validated scoring system (21). In

122 brief, the percentage of TB-associated infiltrations in chest X-rays was assessed and 40 
123 points were added to the score if cavities were present (max. 140 points). An experienced

124 chest physician scored the chest X-rays.

126 Time to sputum culture conversion (TCC) and smear conversion (TSC) were defined as the

127 time (in days) from the initiation of effective anti-TB therapy to the date of the first negative

128 culture or sputum smear (date of collection). Therapy was deemed effective according to 129 DST results.

131 Individualized anti-TB drug regimens for patients with M/XDR-TB were designed using

132 current therapy recommendations, and results of molecular and phenotypic drug

133 susceptibility testing $(10,11,23,24)$. Patients with non-M/XDR TB were treated following 134 national TB guidelines (25).

136 Smear and culture conversion for the first six months after treatment initiation were

137 evaluated using survival analysis, and compared with survival curves for the two cohorts by a

138 logrank test. Kaplan-Meier estimates derived from the survival curves for smear and culture

139 conversion at month 2 and month 6 , and the medium time to smear or culture conversion

140 are reported. Measured clinical score and change in radiological score were assessed by

141 mean and $95 \%$ confidence interval at a priori time points. All statistical tests used a two-

142 sided alpha-value of 0.05 to assess statistical significance. Analyses were performed using

143 STATA (Version 14, StataCorp LLC, College Station, Texas, USA). 
146 Seventy-five patients were enrolled, of whom 46 were infected with non-M/XDR and 29 with

147 M/XDR-TB strains. Patients' characteristics are displayed in table 1. Of the 29 patients with

$148 \mathrm{M} / \mathrm{XDR}-\mathrm{TB}$, eight patients were infected with an XDR strain of $M$. tuberculosis. Median age 149 was higher in patients with non-M/XDR-TB compared to M/XDR-TB patients (43.0 years (IQR $15031.0-58.0$ ) vs. 36.0 years (IQR $30.0-41.0$ )). Sex distribution (non-M/XDR-TB: males 30 151 (65.2\%) vs. M/XDR-TB: males $16(62.1 \%)$ ) and median BMI values (non-M/XDR-TB: 21.1 $152 \mathrm{~kg} / \mathrm{m}^{2}$ (IQR 18.1 - 24.8 vs. M/XDR-TB: $21.7 \mathrm{~kg} / \mathrm{m}^{2}(19.4-25.4)$ ) were similar in both patient 153 groups.

155 The results of available DST results are shown in table 2. Among the 46 patients with non$156 \mathrm{M} / \mathrm{XDR}-\mathrm{TB}$ two patients had isoniazid mono-resistance. No further drug-resistances to first-

157 line drugs (ethambutol and pyrazinamide tested in 44 strains) nor second-line drugs 158 (prothionamide, ofloxacin, and capreomycin tested in 11 strains) were detected in this 159 group. Strains from patients with M/XDR-TB showed high frequencies of first-line drug 160 resistance; 18/27 (66.7\%) for ethambutol, 22/27 (81.5\%) pyrazinamide. Additionally, 161 resistance was present in M/XDR-TB strains to capreomycin 10/29 (34.5\%), amikacin 7/29 162 (24.1\%), ofloxacin 9/27 (33.3\%), prothionamide 14/27 (51.9\%), para-amino-salicylic acid 1/27 $163(3.7 \%)$ and linezolid $1 / 27$ (3.7\%). None of the M/XDR-TB strains were resistant to 164 terizidone/cycloserin.

166 The starting therapy regimens are shown in table 3. Most M/XDR-TB patients receiving 167 fluoroquinolones were treated with moxifloxacin $18 / 22(81.8 \%)$ and only $4 / 22(18.2 \%)$ 168 received levofloxacin. Of 21/29 (72.4\%) M/XDR-TB patients who were treated with second169 line injectable drugs $14 / 21$ (66.7\%) patients were given capreomycin and 7/21 (33.3\%) 
amikacin. Only $3 / 29(10.4 \%)$ M/XDR-TB patients were treated with regimens containing

171 bedaquiline or delamanid.

172 Patients with M/XDR-TB had a slightly lower Kaplan-Meier estimate for smear conversion

173 compared to patients with non-M/XDR-TB at month two ( $78 \%$ versus $90 \%$, respectively), and

174 at month six (93\% and 96\%, respectively, $p=0.004$; table 4 and figure $1 \mathrm{~A}$ ). Such a difference

175 was not seen for culture conversion (figure 1B and table 4), where Kaplan-Meier estimates

176 were $61 \%$ and $67 \%$, respectively at month two, and $95 \%$ and $97 \%$, respectively, at month six

$177(p=0.191)$. The median time to smear conversion was 31 days (IQR: 14-56) and 19 days

(IQR: 10-32) for patient with non-M/XDR-TB and M/XDR-TB respectively. Time to culture conversion was with 39 days, identical in both groups.

The mean clinical scores before treatment initiation were higher in patients with nonM/XDR-TB TB than in patients with M/XDR-TB $(5.8(95 \% \mathrm{Cl} 3.5-7.4)$ vs. $4.8(95 \% \mathrm{Cl} 3.6-5.6))$ with a decline of mean scores in both cohorts after therapy initiation (online appendix table 1 and figure 2). There were no obvious differences in the development of the clinical score between treatment initiation and month 6 .

The radiological extent of disease evaluated using the Ralph score at baseline showed similar values in both groups (mean 58.8 vs. 52.1 points; online appendix table 1 and figure $\mathbf{3}$ ).

187 Although there was a slight increase of pulmonary infiltrations in the non-M/XDR-TB cohort 188 after treatment start, the score values declined over the time of treatment. Here, the 189 radiological scores of patients with M/XDR-TB remained at higher values, which was mainly explained by a higher frequency of the persistence of cavitary lesions in the chest X-rays. A

191 higher proportion of patients with M/XDR-TB previously had TB and thus prior lung damage, which would explain more extensive infiltrations. 


\section{Discussion}

194 We compared early treatment response in a prospective cohort of patients with M/XDR-TB

195 and patients with non-M/XDR-TB in Germany, a country where unrestricted diagnostic and

196 therapeutic resources for the management of patients with M/XDR-TB are available. Six-

197 months culture conversion was similar for patients with non-MDR-TB and M/XDR-TB, which

198 could suggest a high chance of cure for patients with M/XDR-TB.

199

200 Our findings are in line with published data showing that the 6-months culture conversion

201 status is indicative for sustained treatment response in patients with M/XDR-TB $(14,26)$.

202 Using 6-months culture conversion status and a one-year follow-up after therapy completion

203 as markers for therapy outcome has also been suggested in a recently published study (14).

204 Although the 2-months culture conversion status has been evaluated as a surrogate for 205 treatment response in clinical trials evaluating novel anti-TB drugs, such as bedaquiline and 206 delamanid $(27,28)$, treatment outcomes are better correlated to the 6-months culture 207 conversion status (29). Six-months culture-conversion status was similar in both groups

208 (table 4). In the present study, the 2-months culture conversion rates of our M/XDR-TB 209 patients undergoing tailored treatment regimens on the basis of comprehensive drug 210 susceptibility testing exceeded the rates from trials presenting promising novel drugs with 211 excellent therapy results $(27,28,30)$.

213 Standardized therapy regimens as presented by the "Bangladesh" regimen have yielded 214 excellent outcomes in specific settings with low frequencies of second-line drug resistance 215 (30). Based on these results the WHO made a conditional recommendation for a "shorter 216 course regimen" for the treatment of MDR-TB (9). However, only very few patients from the 217 European region may be eligible for this regimen due to high frequencies of second-line drug 
218 resistance $(13,31,32)$, which may lead to treatment failure and acquisition of additional

219 drug resistance (33). The low eligibility of patients from this setting is also reflected by our

220 study where high frequencies of first and second-line drug resistance were identified. This

221 strongly indicates that standard treatment regimens could lead to the emergence of

222 additional drug resistance due to inadequate therapy.

224 This study also showed that tailored treatment regimens in our study were highly variable. In

225 fact, such individualized therapy regimens were shown to lead to very high frequencies of 226 favorable treatment outcomes in an Austrian cohort (15). In contrast to patients who 227 received standardized treatment regimens, higher frequencies of cure for patients with 228 MDR-TB from the European region were found with individualized treatment regimens in a 229 large European multicenter cohort (Günther et al. submitted). Higher frequencies of 230 treatment success in patients receiving individualized therapy were also shown by large 231 meta-analyses comparing treatment results of patients with MDR-TB under standardized or 232 individualized regimens $(16,34)$.

234 We also evaluated the performance of an existing radiological score using chest X-rays and a 235 novel clinical score to further characterize the effect of treatment on an individual basis (21, 236 35). The items included in the clinical score were based on a published scoring system, which 237 was shown to predict mortality and treatment response in African cohorts $(22,36,37)$. 238 Although we were able to show declining clinical and radiological scores after therapy 239 initiation (figure 3 and 4, online appendix table 1), our intention to correlate the scores' 240 trajectories with the established markers such as time to culture or to smear conversion, and 241 2- or 6 month culture conversion status failed, given the almost uniformly high frequency of 242 conversion in a relatively small cohort. Unfortunately, the small number of patients 
243 precluded data reduction strategies like principal component analysis to evaluate the clinical

244 score. The decline in radiological score observed in our study was slow probably due to 245 persistence of cavitary lesions, which are slower to resolve, and in the presence of an 246 adequate clinical and microbiological response. Nevertheless, these or other clinically 247 derived scores may serve as alternative end-points for future biomarker validation and 248 should be reconsidered in future studies (38).

249

Although the relatively low number of patients evaluated limits our study, a very close 251 microbiological and clinical monitoring and the observations from clinical and radiological 252 scores strengthens our findings.

253

254 In conclusion, under optimal clinical conditions with availability of novel diagnostics and 255 individualized therapy, patients with M/XDR-TB can achieve 6-months culture conversion, 256 the frequency of which is similar to that of to patients with non-M/XDR-TB. This personalized 257 approach to therapy may have the potential to yield high frequencies of cure. The clinical 258 and radiological scores should be further evaluated with the aim to identify and validate 259 markers to individualize the duration of therapy.

\section{Acknowledgements}

262 We thank Cordula Ehlers, Jessica Hofmeister, Franziska Daduna, Nelleke Smitsmann, and 263 Dagmar Schaub for the study management and data entry. The study is funded by the 264 German Center for Infection Research (DZIF). 
266 Table 1. Clinical characteristics of patients with non-MDR-TB and M/XDR-TB.

\begin{tabular}{|c|c|c|c|c|c|c|}
\hline & \multicolumn{2}{|c|}{ Non-M/XDR* } & \multicolumn{2}{|c|}{$\mathrm{M} / \mathrm{XDR}$} & \multicolumn{2}{|l|}{ Total } \\
\hline & \multicolumn{2}{|c|}{$N=46$} & \multicolumn{2}{|c|}{$N=29$} & \multicolumn{2}{|c|}{$N=75$} \\
\hline & $\mathrm{n}$ & $\%$ & $\mathrm{n}$ & $\%$ & $\mathrm{n}$ & $\%$ \\
\hline \multicolumn{7}{|l|}{ Sex } \\
\hline Male & 30 & 65.2 & 18 & 62.1 & 48 & 64.0 \\
\hline Age, median (IQR) & 43.0 & $31.0-58.0$ & 36.0 & $30.0-41.0$ & 39.0 & $31.0-56.0$ \\
\hline BMI, median (IQR) & 21.1 & $18.1-24.8$ & 21.7 & $19.4-25.4$ & 21.2 & $18.3-25.4$ \\
\hline \multicolumn{7}{|l|}{ TB contact } \\
\hline No & 21 & 45.7 & 9 & 31.0 & 30 & 40.0 \\
\hline Yes & 6 & 13.0 & 4 & 13.8 & 10 & 13.3 \\
\hline missing & 19 & 41.3 & 16 & 55.2 & 35 & 46.7 \\
\hline \multicolumn{7}{|l|}{ BCG } \\
\hline Yes & 13 & 28.3 & 16 & 55.2 & 29 & 38.7 \\
\hline missing & 28 & 60.9 & 12 & 41.4 & 40 & 53.3 \\
\hline \multicolumn{7}{|l|}{ TB type } \\
\hline not previously treated for TB & 35 & 76.1 & 18 & 62.1 & 53 & 70.7 \\
\hline Relapse & 6 & 13.0 & 6 & 20.7 & 12 & 16.0 \\
\hline Failure & 0 & 0.0 & 1 & 3.4 & 1 & 1.3 \\
\hline Return from default & 1 & 2.2 & 1 & 3.4 & 2 & 2.7 \\
\hline missing & 4 & 8.7 & 3 & 10.3 & 7 & 9.3 \\
\hline \multicolumn{7}{|l|}{ Previous TB } \\
\hline Yes & 7 & 15.2 & 10 & 34.5 & 17 & 22.7 \\
\hline missing & 14 & 30.4 & 4 & 13.8 & 18 & 24.0 \\
\hline \multicolumn{7}{|l|}{ Diabetes } \\
\hline Yes & 4 & 8.7 & 2 & 6.9 & 6 & 8.0 \\
\hline missing & 19 & 41.3 & 5 & 17.2 & 24 & 32.0 \\
\hline \multicolumn{7}{|l|}{ Hepatitis B } \\
\hline Yes & 3 & 6.5 & 1 & 3.4 & 4 & 5.3 \\
\hline missing & 25 & 54.3 & 12 & 41.4 & 37 & 49.3 \\
\hline \multicolumn{7}{|l|}{ Hepatitis C } \\
\hline Yes & 2 & 4.3 & 3 & 10.3 & 5 & 6.7 \\
\hline missing & 25 & 54.3 & 11 & 37.9 & 36 & 48.0 \\
\hline
\end{tabular}


Table 2. Results of Mycobacterium tuberculosis phenotypic drug susceptibility testing from

270 patients with non-M/XDR and $M / X D R$.

\begin{tabular}{|c|c|c|c|c|c|c|c|c|}
\hline & \multicolumn{4}{|c|}{ Non M/XDR* } & \multicolumn{4}{|c|}{ M/XDR } \\
\hline & \multicolumn{4}{|c|}{$N=46$} & \multicolumn{4}{|c|}{$N=29$} \\
\hline \multirow[t]{2}{*}{ Drug } & \multicolumn{2}{|c|}{ Tested } & \multicolumn{2}{|c|}{ Resistant } & \multicolumn{2}{|c|}{ Tested } & \multicolumn{2}{|c|}{ Resistant } \\
\hline & $\mathrm{N}$ & $\%$ & $\mathrm{~N}$ & $\%$ & $\mathrm{~N}$ & $\%$ & $\mathrm{~N}$ & $\%$ \\
\hline $\mathrm{H}$ & 45 & 97.8 & 2 & 4.4 & 27 & 93.1 & 27 & 100 \\
\hline$R$ & 46 & 100 & 0 & 0.0 & 29 & 100 & 29 & 100 \\
\hline$E$ & 44 & 95.7 & 0 & 0.0 & 27 & 93.1 & 18 & 66.7 \\
\hline$Z$ & 44 & 95.7 & & & 27 & 93.1 & 22 & 81.5 \\
\hline $\mathrm{L}$ & & & & & 10 & 34.5 & 9 & 90.0 \\
\hline$M$ & & & & & 16 & 55.2 & 9 & 56.3 \\
\hline 0 & 11 & 23.9 & 0 & 0.0 & 27 & 93.1 & 9 & 33.3 \\
\hline$S$ & & & & & 16 & 55.2 & 12 & 75.0 \\
\hline$A$ & & & & & 29 & 100 & 7 & 24.1 \\
\hline $\mathrm{C}$ & 11 & 23.9 & 0 & 0.0 & 29 & 100 & 10 & 34.5 \\
\hline$K$ & & & & & 8 & 27.6 & 2 & 25.0 \\
\hline$P$ & 11 & 23.9 & 0 & 0.0 & 27 & 93.1 & 14 & 51.9 \\
\hline$T$ & & & & & 26 & 89.7 & 0 & 0.0 \\
\hline c & & & & & 1 & 3.4 & 1 & 100 \\
\hline 1 & & & & & 27 & 93.1 & 1 & 3.7 \\
\hline$p$ & & & & & 27 & 93.1 & 1 & 3.7 \\
\hline $\mathrm{m}$ & & & & & 1 & 3.4 & 1 & 100 \\
\hline$a$ & & & & & 1 & 3.4 & 1 & 100 \\
\hline
\end{tabular}

$271 * 44$ patients with pan-drug susceptible TB and 2 patients with isoniazid mono-resistance

272 H: isoniazid; R: rifampicin; Z: pyrazinamid; E: ethambutol; O: ofloxacin; M: moxifloxacin; L: levofloxacin; S: 273 streptomycin; C: capreomycin; A: amikacin; K: kanamycin; P: protionamide; T: terizidone/cycloserin; I: linezolid;

274 c: clofazamin; p: para-amino-salicylic acid; m: meropenem; a: amoxicillin/clavulanic acid. 
Table 3. Starting regimens in patients with non-MDR-TB and M/XDR-TB.

\begin{tabular}{|c|c|c|c|c|c|c|c|c|c|c|c|}
\hline \multicolumn{10}{|c|}{ Drugs } & \multirow[t]{2}{*}{ Non-M/XDR* } & \multirow[t]{2}{*}{$\mathrm{M} / \mathrm{XDR}$} \\
\hline HRZE & - & OML & - & SCAK & - & PTlc & - & $\mathrm{BD}$ & - pma & & \\
\hline XXXX & - & $\ldots$ & - & $\ldots$ & - & $\ldots$ & - & $\ldots$ & $-\ldots$ & 34 & 0 \\
\hline $\mathrm{XXXX}$ & - & $\ldots$ & - & $\ldots$ & - & ..X. & - & . & $-X$. & 1 & 0 \\
\hline XXX. & - & $\ldots$ & - & $\ldots$ & - & $\ldots$ & - & . & $-\ldots$ & 6 & 0 \\
\hline $\mathrm{XX} . \mathrm{X}$ & - & $\ldots$ & - & $\mathrm{X} \ldots$ & - & $\ldots$ & - & . & $-\ldots$ & 2 & 0 \\
\hline $\mathrm{XX} . \mathrm{X}$ & - & $\ldots$ & - & $\cdots$ & - & $\ldots$ & - & .. & $\cdots$ & 1 & 0 \\
\hline$X . . X$ & - & ... & - & $\ldots$ & - & $\ldots$ & - & $\cdots$ & $\cdots$ & 1 & 0 \\
\hline. $\mathrm{XXX}$ & - &.$X$ & - & $\ldots$ & - & $\ldots$ & - & . & ... & 1 & 0 \\
\hline .X.. & - &.$X$ & - &.$X \ldots$ & - & XX.. & - & $\ldots$ & $\ldots$ & 0 & 1 \\
\hline . XX & - &.$X$. & - & .X.. & - & XX.. & - & $\ldots$ & $\ldots$ & 0 & 1 \\
\hline$\ldots X X$ & - &.$X$. & - &.$X \ldots$ & - & XX.. & - & $\ldots$ & $\ldots$ & 0 & 1 \\
\hline. $\mathrm{XX}$ & - &. $\mathrm{X}$ & - &..$X$. & - & XX .. & - & $\cdots$ & $-\ldots$ & 0 & 1 \\
\hline. $\mathrm{XX}$ & - &..$X$ & - & . X. & - &. $\mathrm{XX}$ & - & .. & $-\ldots$ & 0 & 1 \\
\hline . X. & - &.$X$. & - & . X.. & - & $\mathrm{XXX}$ & - & $\cdots$ & $-\ldots$ & 0 & 1 \\
\hline . .X. & - &.$X$. & - & .X.. & - & XX.. & - & $\ldots$ & $-\ldots$ & 0 & 2 \\
\hline ..X. & - &.$X$ & - & .X.. & - &.$X \ldots$ & - & $\ldots$ & $-\ldots$ & 0 & 1 \\
\hline . .X. & - &.$X$ & - & ..X. & - & . .XX & - & $\ldots$ & $-\ldots$ & 0 & 1 \\
\hline . .X. & - & $\ldots X$ & - & $\ldots X$ & - &. $\mathrm{XXX}$ & - & $\ldots$ & $-\ldots$ & 0 & 1 \\
\hline . X . & - & $\cdots$ & - & . X.. & - & . XX. & - & $\cdots$ & $-\ldots$ & 0 & 1 \\
\hline ..X. & - & . . & - & . . X. & - & .X.X & - & .. & $-\mathrm{XXX}$ & 0 & 1 \\
\hline . X. & - & $\cdots$ & - & $\ldots$ & - & .X.X & - & .. & $-\mathrm{XXX}$ & 0 & 1 \\
\hline ...X & - &.$X$ & - & . X.. & - & XX.X & - & .. & $-\ldots$ & 0 & 1 \\
\hline$\ldots X$ & - &.$X$ & - & .X.. & - & XX .. & - & $\ldots$ & $-\ldots$ & 0 & 1 \\
\hline$\ldots X$ & - &.$X$ & - & . X... & - & X... & - & .. & $-\ldots$ & 0 & 1 \\
\hline$\ldots X$ & - & $\ldots X$ & - & $\ldots$ & - & XXX. & - & $\ldots$ & $-\ldots$ & 0 & 1 \\
\hline$\ldots$ & - &. $\mathrm{X}$ & - & . X.. & - & XXX & - & $\ldots$ & $-X \ldots$ & 0 & 1 \\
\hline$\ldots \mathrm{X}$ & - & ... & - & . . X. & - & .XX. & - & $\mathrm{X}$. & $-\ldots$ & 0 & 1 \\
\hline$\ldots$ & - &.$X$. & - & . X.. & - & .XX. & - & .. & $-X \ldots$ & 0 & 1 \\
\hline ... & - &.$X$ & - & . X.. & - & . X.. & - & $\ldots$ & $-X \ldots$ & 0 & 1 \\
\hline$\ldots$ & - &.$X$ & - & ..X. & - &.$X X$ & - & $\ldots$ & $-X \ldots$ & 0 & 1 \\
\hline$\ldots$ & - &.$X$ & - & $\ldots$ & - & XXXX & - & $\ldots$ & $-\ldots$ & 0 & 1 \\
\hline$\ldots$ & - & $\ldots X$ & - & $\ldots$ & - &.$X X$ & - & $\mathrm{X}$ & $-\ldots$ & 0 & 1 \\
\hline$\ldots$ & - & $\ldots$ & - & ... & - & . XXX & - & $\mathrm{X}$. &.$- X X$ & 0 & 2 \\
\hline$\ldots$ & - & $\cdots$ & - & $\ldots \ldots$ & - & XXX & - & $\ldots$ & $-\mathrm{XXX}$ & 0 & 1 \\
\hline ... & - & $\ldots$ & - & ... & - & XX & - & $\ldots$ & $-\ldots$ & 0 & 1 \\
\hline
\end{tabular}

*44 patients with pan-drug susceptible TB and 2 patients with isoniazid mono-resistance

278 "." indicates if drug was not used and " $\mathrm{X}$ " if drug was used; H: isoniazid; R: rifampicin; Z: pyrazinamid; E: 279 ethambutol; O: ofloxacin; M: moxifloxacin; L: levofloxacin; S: streptomycin; C: capreomycin; A: amikacin; K: 280 kanamycin; P: protionamide; T: terizidone/cycloserin; I: linezolid; c: clofazamin; B: bedaquiline; D: delamanid; p: 281 PAS; m: meropenem; a: amoxicillin/clavulanic acid. 
284 Table 4. Percentage of patients with M. tuberculosis culture and smear conversion at month 2852 and 6 after therapy initiation and median time to conversion with interquartile range for 286 patients with non-M/XDR-TB and M/XDR-TB.

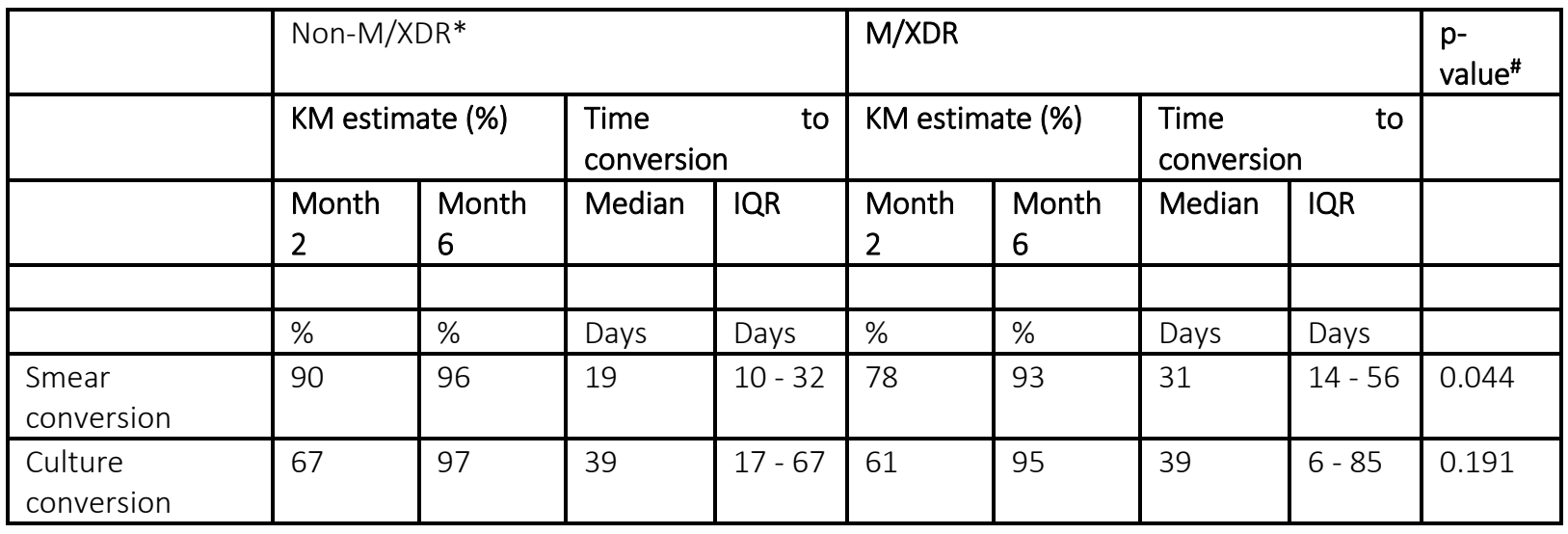

$287 * 44$ patients with pan-drug susceptible TB and 2 patients with isoniazid mono-resistance 288 \#Derived from Log-rank test. 


\section{FIGURES}

291 A

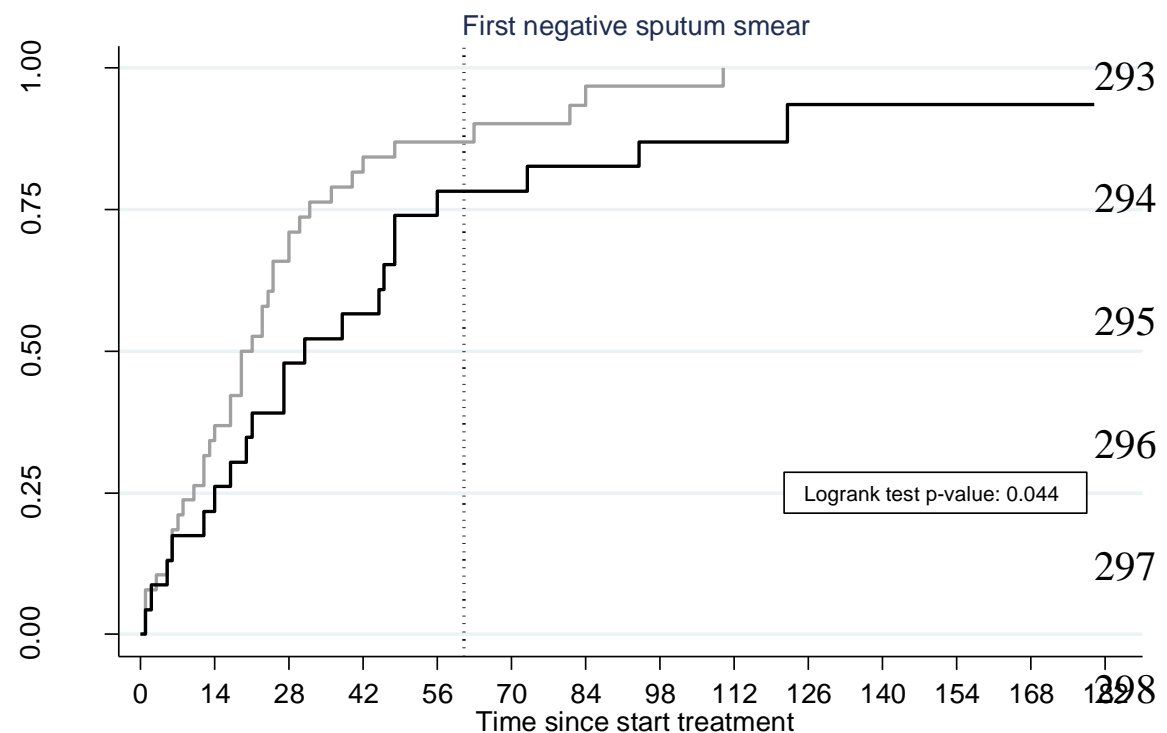

Number at risk

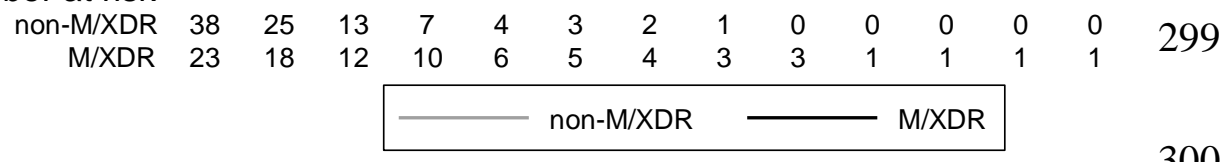

300

301

B

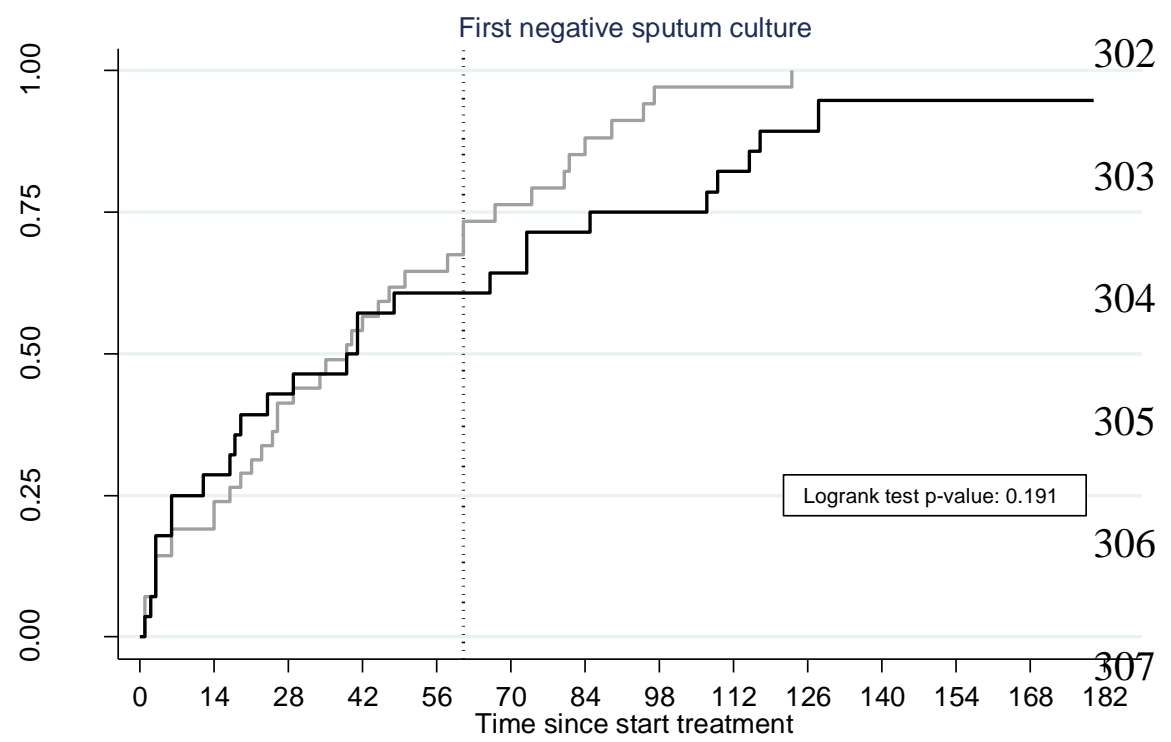

Number at risk

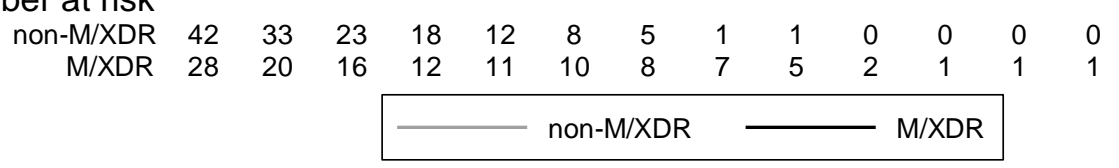

308 
310 Figure 1A and 1B.

311 A. Kaplan-Meier estimates for the first negative sputum smear microscopy in patients with

312 non-M/XDR-TB (grey line) and M/XDR-TB (black line) after therapy initiation. Below the X-

313 axis, the number at risk is shown. B. Kaplan-Meier estimates for the first negative sputum

314 culture (liquid and solid) in patients with non-M/XDR-TB (grey line) and M/XDR-TB (black

315 line) after therapy initiation. Below the $\mathrm{X}$-axis, the number at risk is shown. 


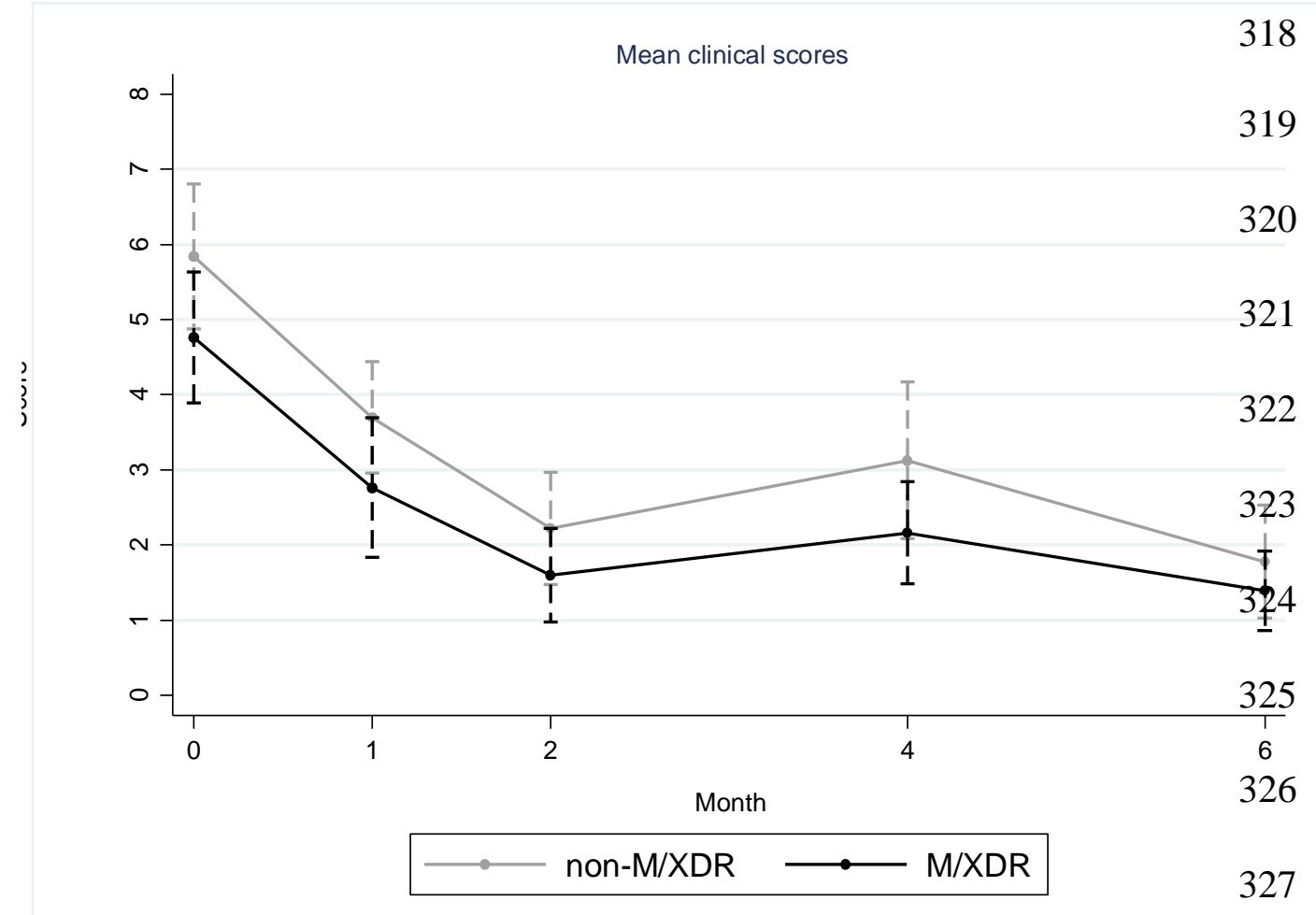

329 Figure 2. Mean clinical scores ( $\mathrm{Y}$-axis) during the course of therapy (X-axis, months) for

330 patients with susceptible (gray line) and M/XDR-TB (black line) with $95 \%$ confidence interval 331 (dashed lines).

332 


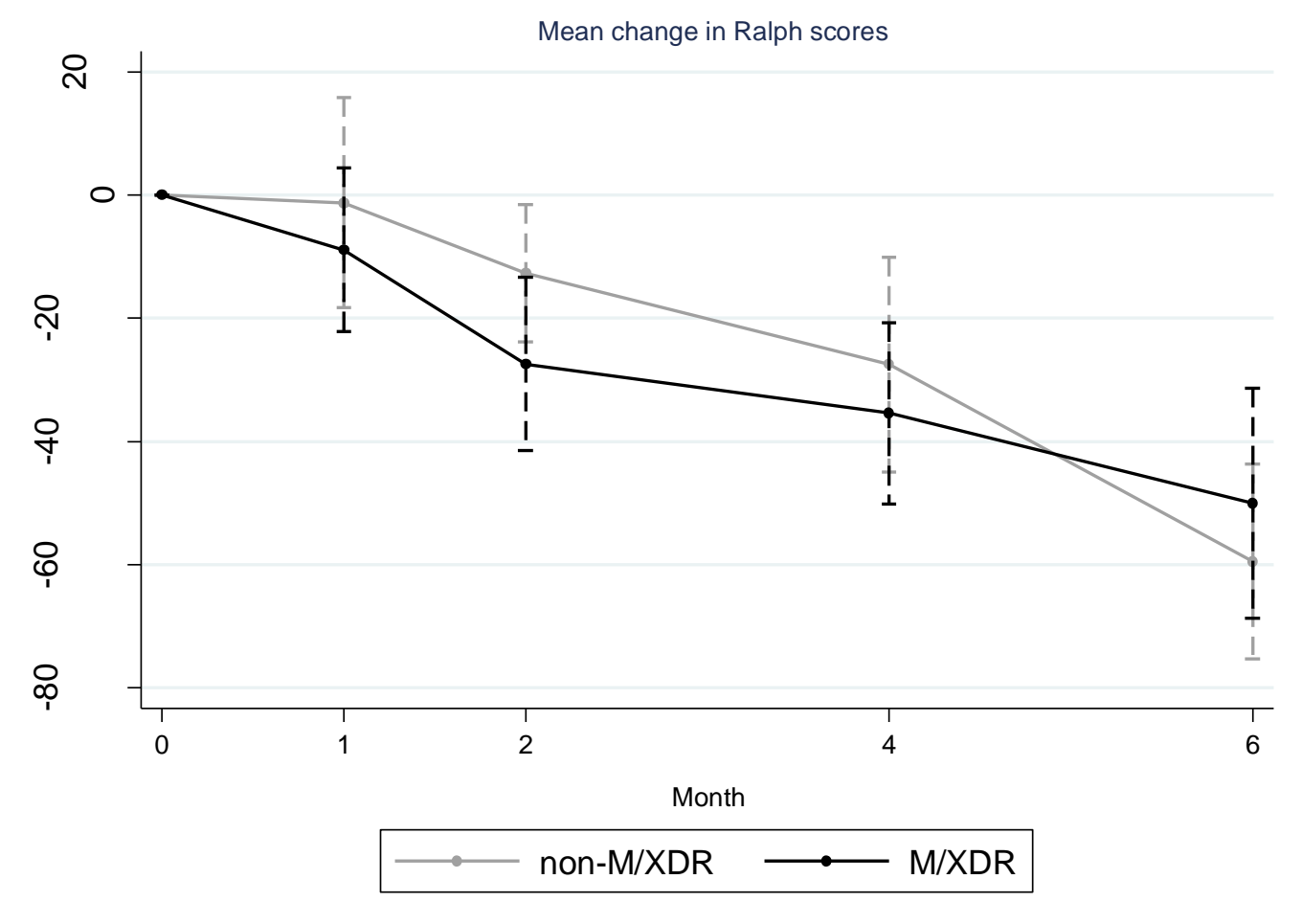

333

334 Figure 3. Mean change of radiological (Ralph) scores (Y-axis, \%) in the course of therapy (X-

335 axis, months) for patients with susceptible (grey line) and M/XDR-TB (black line) with 95\%

336 confidence interval (dashed lines).

337 
340 1. World Health Organization. Global tuberculosis report 2016. Geneva, 341 Switzerland2016.

342 2. Global Burden Diseases Study- Mortality Causes of Death Collaborators. Global, regional, and national life expectancy, all-cause mortality, and cause-specific mortality for 249 causes of death, 1980-2015: a systematic analysis for the Global Burden of Disease Study 2015. Lancet. 2016;388(10053):1459-544.

346 3. European Centre for Disease Prevention and Control (ECDC). Tuberculosis surveillance and monitoring in Europe 2016. Stockholm, Sweden: European Centre for Disease Prevention and Control, 2017.

4. Ahuja SD, Ashkin D, Avendano M, Banerjee R, Bauer M, Bayona JN, et al. Multidrug resistant pulmonary tuberculosis treatment regimens and patient outcomes: an individual patient data meta-analysis of 9,153 patients. PLoS Med. 2012;9(8):e1001300.

352 5. Falzon D, Gandhi N, Migliori GB, Sotgiu G, Cox HS, Holtz TH, et al. Resistance to fluoroquinolones and second-line injectable drugs: impact on multidrug-resistant $T B$ outcomes. Eur Respir J. 2013;42(1):156-68.

6. Sotgiu G, Centis R, D'Ambrosio L, Alffenaar JW, Anger HA, Caminero JA, et al. Efficacy, safety and tolerability of linezolid containing regimens in treating MDR-TB and XDR-TB: systematic review and meta-analysis. Eur Respir J. 2012;40(6):1430-42.

358 7. Günther G, Gomez GB, Lange C, Rupert S, van Leth F, Tbnet. Availability, price and

359 affordability of anti-tuberculosis drugs in Europe: a TBNET survey. Eur Respir J. $360 \quad 2015 ; 45(4): 1081-8$.

361 8. G20 Leaders' Declaration. Shaping an interconnected world. In: G20, editor. 362 Hamburg2017.

363 9. World Health Organization. Rapid diagnostic test and shorter, cheaper treatment signal new hope for multidrug-resistant tuberculosis patients Geneva, Switzerland2016 [cited 2016 29.7.2016]. Available from:

366 http://www.who.int/mediacentre/news/releases/2016/multidrug-resistant-tuberculosis/en/.

367 10. World Health Organization. Guidelines for the programmatic management of drug368 resistant tuberculosis 2016 update. Geneva, Switzerland: WHO Library Cataloguing-in369 Publication Data; 2016.

370 11. Lange C, Abubakar I, Alffenaar JW, Bothamley G, Caminero JA, Carvalho AC, et al. 371 Management of patients with multidrug-resistant/extensively drug-resistant tuberculosis in 372 Europe: a TBNET consensus statement. Eur Respir J. 2014;44(1):23-63.

373 12. Balabanova Y, Fiebig L, Ignatyeva O, Riekstina V, Danilovits $M$, Jaama K, et al. 374 Multidrug-resistant TB in Eastern region of the EU: is the shorter regimen an exception or a 375 rule? Thorax. 2017.

376 13. Lange $C$, Duarte R, Frechet-Jachym M, Günther G, Guglielmetti L, Olaru ID, et al. 377 Limited Benefit of the New Shorter Multidrug-Resistant Tuberculosis Regimen in Europe. Am 378 J Respir Crit Care Med. 2016;194(8):1029-31.

379 14. Günther G, Lange C, Alexandru S, Altet N, Avsar K, Bang D, et al. Treatment Outcomes 380 in Multidrug-Resistant Tuberculosis. N Engl J Med. 2016;375(11):1103-5.

381 15. Olaru ID, Lange C, Indra A, Meidlinger L, Huhulescu S, Rumetshofer R. High Rates of 382 Treatment Success in Pulmonary Multidrug-Resistant Tuberculosis by Individually Tailored 383 Treatment Regimens. Ann Am Thorac Soc. 2016;13(8):1271-8.

384 16. Bastos ML, Lan Z, Menzies D. An updated systematic review and meta-analysis for 385 treatment of multidrug-resistant tuberculosis. Eur Respir J. 2017;49(3). 
17. Olaru ID, Lange C, Heyckendorf J. Personalized medicine for patients with MDR-TB. J Antimicrob Chemother. 2016;71(4):852-5. 18. Olaru ID, von Groote-Bidlingmaier F, Heyckendorf J, Yew WW, Lange C, Chang KC. Novel drugs against tuberculosis: a clinician's perspective. Eur Respir J. 2015;45(4):1119-31. 19. Pankhurst $\amalg$, del Ojo Elias C, Votintseva AA, Walker TM, Cole K, Davies J, et al. Rapid, comprehensive, and affordable mycobacterial diagnosis with whole-genome sequencing: a prospective study. The Lancet Respiratory Medicine. 2016;4(1):49-58.

393 20. Votintseva AA, Bradley P, Pankhurst L, Del Ojo Elias C, Loose M, Nilgiriwala K, et al. Same-Day Diagnostic and Surveillance Data for Tuberculosis via Whole-Genome Sequencing of Direct Respiratory Samples. J Clin Microbiol. 2017;55(5):1285-98.

21. Ralph AP, Ardian M, Wiguna A, Maguire GP, Becker NG, Drogumuller G, et al. A simple, valid, numerical score for grading chest $x$-ray severity in adult smear-positive pulmonary tuberculosis. Thorax. 2010;65(10):863-9. al. The Bandim tuberculosis score: reliability and comparison with the Karnofsky performance score. Scand J Infect Dis. 2013;45(4):256-64.

23. Horsburgh CR, Jr., Barry CE, 3rd, Lange C. Treatment of Tuberculosis. N Engl J Med. 2015;373(22):2149-60.

24. World Health Organization. Companion handbook to the WHO guidelines for the programmatic management of drug-resistant tuberculosis. Geneva, Switzerland 2014. 25. Schaberg T, Bauer T, Castell S, Dalhoff K, Detjen A, Diel R, et al. [Recommendations for therapy, chemoprevention and chemoprophylaxis of tuberculosis in adults and children. German Central Committee against Tuberculosis (DZK), German Respiratory Society (DGP)]. Pneumologie. 2012;66(3):133-71.

410 26. Kurbatova EV, Cegielski JP, Lienhardt C, Akksilp R, Bayona J, Becerra MC, et al. Sputum culture conversion as a prognostic marker for end-of-treatment outcome in patients with multidrug-resistant tuberculosis: a secondary analysis of data from two observational cohort studies. Lancet Respir Med. 2015;3(3):201-9.

27. Gler MT, Skripconoka V, Sanchez-Garavito E, Xiao H, Cabrera-Rivero JL, VargasVasquez DE, et al. Delamanid for multidrug-resistant pulmonary tuberculosis. N Engl J Med. 2012;366(23):2151-60.

28. Diacon AH, Pym A, Grobusch MP, de los Rios JM, Gotuzzo E, Vasilyeva I, et al. Multidrug-resistant tuberculosis and culture conversion with bedaquiline. N Engl J Med. 2014;371(8):723-32.

29. Diacon AH, Van Baelen B, Theeuwes M. More on Treatment Outcomes in MultidrugResistant Tuberculosis. N Engl J Med. 2016;375(26):2609-10.

30. Van Deun A, Maug AK, Salim MA, Das PK, Sarker MR, Daru P, et al. Short, highly effective, and inexpensive standardized treatment of multidrug-resistant tuberculosis. Am J Respir Crit Care Med. 2010;182(5):684-92.

31. Günther G, van Leth F, Altet N, Dedicoat M, Duarte R, Gualano G, et al. Beyond multidrug-resistant tuberculosis in Europe: a TBNET study. Int J Tuberc Lung Dis. 2015;19(12):1524-7.

32. Gunther G, van Leth F, Alexandru S, Altet N, Avsar K, Bang D, et al. Multidrugresistant tuberculosis in Europe, 2010-2011. Emerg Infect Dis. 2015;21(3):409-16. resistance and its effect on treatment outcome in patients treated with Standard ShortCourse Chemotherapy for tuberculosis. BMC Pulm Med. 2016;16:26. 
433 34. Orenstein EW, Basu S, Shah NS, Andrews JR, Friedland GH, Moll AP, et al. Treatment 434 outcomes among patients with multidrug-resistant tuberculosis : systematic review and 435 meta-analysis. The Lancet Infectious Diseases. 2009;9(3):153-61.

436 35. Pinto LM, Pai M, Dheda K, Schwartzman K, Menzies D, Steingart KR. Scoring systems 437 using chest radiographic features for the diagnosis of pulmonary tuberculosis in adults: a 438 systematic review. Eur Respir J. 2013;42(2):480-94.

439 36. Rudolf F, Lemvik G, Abate E, Verkuilen J, Schon T, Gomes VF, et al. TBscore II: refining 440 and validating a simple clinical score for treatment monitoring of patients with pulmonary 441 tuberculosis. Scand J Infect Dis. 2013;45(11):825-36.

442 37. Rudolf F, Wagner AJ, Back FM, Gomes VF, Aaby P, Ostergaard L, et al. Tuberculosis 443 case finding and mortality prediction: added value of the clinical TBscore and biomarker 444 suPAR. Int J Tuberc Lung Dis. 2017;21(1):67-72.

445 38. Heyckendorf J, Olaru ID, Ruhwald M, Lange C. Getting personal perspectives on 446 individualized treatment duration in multidrug-resistant and extensively drug-resistant 447 tuberculosis. Am J Respir Crit Care Med. 2014;190(4):374-83. 